\title{
Les accidents vasculaires cérébraux ischémiques après chirurgie vasculaire
}

\author{
Ischemic cerebrovascular accidents after vascular surgery
}

\author{
Mustapha Alilou, MD · Ahmed El Hijri, PhD • \\ Abderrahim Azzouzi, PhD
}

Received: 14 January 2009/Revised: 9 February 2009/ Accepted: 11 February 2009/Published online: 7 March 2009

(c) Canadian Anesthesiologists' Society 2009

\section{Au rédacteur en chef,}

En postopératoire, la survenue d'un accident vasculaire cérébral (AVC) ischémique constitue un événement dramatique avec une mortalité avoisinant les $26 \%{ }^{1}$ Les chirurgies cardiaque, neurologique et vasculaire sont réputées les plus pourvoyeuses de ce type de complication. En effet, l'incidence en chirurgie générale n'est que de 0,08 à $0,7 \%$, alors qu'elle atteint $8,7 \%$ en chirurgie vasculaire. ${ }^{2}$ Les facteurs de risque de survenue de l'AVC postopératoire sont multiples, mais leur part respective est difficile à déterminer à cause du faible effectif des différentes séries publiées. Pour faire l'état des lieux dans notre institution, nous avons réalisé après accord du comité d'éthique, une étude rétrospective sur cinq ans (janvier 2003 à décembre 2007) portant sur tous les malades opérés pour chirurgie vasculaire, à l'hôpital Ibn Sina de Rabat et ayant présenté un AVC ischémique postopératoire.

Sur un ensemble de 497 malades ayant subi une chirurgie vasculaire, 12 ont subi un AVC, soit une incidence de $2,4 \%$. Il s'agit de huit hommes et quatre femmes d'âge moyen de $65 \pm 8$ ans, répartis à parts égales entre chirurgie carotidienne, chirurgie de l'aorte abdominale et chirurgie vasculaire périphérique des membres inférieurs, soit une incidence respective de $4 \%$ (4/100), 2,3\% (4/174) et 1,8\% (4/223). Les antécédents et les incidents périopératoires survenus chez ces patients sont représentés dans le Tableau 1. Les patients de chirurgie carotidienne étaient opérés sous double bloc cervical, alors que les autres patients étaient opérés sous anesthésie générale. La durée d'intervention était en moyenne de $184 \pm 26 \mathrm{~min}$.

M. Alilou, MD ( $\varangle)$ · A. El Hijri, PhD · A. Azzouzi, PhD

Hôpital Ibn Sina, Rabat, Morocco

e-mail: aliloumust@yahoo.fr
Chez la moitié des malades, l'AVC est survenu durant les 24 premières heures postopératoires. Il était sylvien profond dans dix cas, occipital dans deux cas et était responsable d'un coma plus ou moins profond chez un tiers des malades. Neuf malades avaient une hémiplégie ou une hémiparésie, un malade une monoparésie brachiale et deux malades une cécité bilatérale. Cinq patients ont présenté des convulsions. Cinq patients (42\%) sont décédés; les autres ont gardé des séquelles plus ou moins importantes.

La revue de la littérature montre que la genèse de l'AVC postopératoire est multifactorielle, associant des facteurs en rapport avec le terrain, la chirurgie ou l'anesthésie. Ainsi, les antécédents d'AVC constitué ou transitoire et la présence d'une sténose carotidienne symptomatique constituent des facteurs prédictifs majeurs. ${ }^{3}$ Ce risque n'est pas démontré en cas de sténose asymptomatique. La dysfonction systolique du ventricule gauche augmente le risque d'AVC, particulièrement en cas d'association d'une arythmie complète par fibrillation auriculaire ou d'arrêt préopératoire d'un traitement anticoagulant ou antiagrégant plaquettaire. ${ }^{3,4}$ Les événements hémodynamiques périopératoires (hypertension et surtout collapsus cardiovasculaire) sont souvent incriminés. ${ }^{2}$ Cependant, en chirurgie carotidienne, la majorité des AVC sont imputables à des accidents emboliques, essentiellement dus à la manipulation chirurgicale, et sont controlatéraux à la lésion carotidienne ou bilatéraux. ${ }^{4}$ Les AVC d'origine hémodynamique, quant à eux, sont plus tardifs et sont favorisés par l'hypovolémie et les pertes sanguines non compensées. Le type d'anesthésie semble également jouer un rôle. Ainsi, en chirurgie carotidienne, le risque est plus élevé avec l'anesthésie générale qu'avec l'anesthésie locorégionale. ${ }^{2}$ Le siège de l'AVC est dans la plupart des cas sylvien, témoignant de l'obstruction des artères carotides plutôt que de l'atteinte vertébrobasilaire. Quand l'infarctus sylvien est 
Tableau 1 Antécédents et incidents périopératoires

Nombre (\%)

\begin{tabular}{ll} 
Antécédents & \\
Hypertension artérielle & $3(25)$ \\
Fibrillation auriculaire & $2(16,7)$ \\
Diabète & $3(25)$ \\
AVCI & $2(16,7)$ \\
Tabac & $6(50)$ \\
Incidents périopératoires & \\
Hypertension peropératoire & $5(41,7)$ \\
Hypotension peropératoire & $3(25)$ \\
Choc hémorragique postopératoire & $3(25)$ \\
Choc cardiogénique postopératoire & $2(16,7)$ \\
\hline
\end{tabular}

$A V C I$ accident vasculaire cérébral ischémique

étendu, la mortalité peut atteindre $65 \%$, alors que les infarctus vertébro-basilaires ont un meilleur pronostic. Les infarctus profonds de faible volume et les infarctus lacunaires ont également un bon pronostic immédiat avec une mortalité inférieure à 5\%, mais un risque élevé de récidive et de décès d'origine cardiaque. ${ }^{5}$

Conflit d'intérêt Aucun.

\section{Références}

1. Parikh S, Cohen JR. Perioperative stroke after general surgical procedures. NY State J Med 1993; 93: 162-5.

2. Selim M. Perioperative stroke. N Engl J Med 2007; 356: 706-13.

3. Limburg $M$, Wijdicks $E F, L i H$. Ischemic stroke after surgical procedures: clinical features, neuroimaging, and risk factors. Neurology 1998; 50: 895-901.

4. Epstein AE, Alexander JC, Gutterman DD, Maisel W, Wharton JM; American College of Chest Physicians. Anticoagulation: American College of Chest Physicians guidelines for the prevention and management of postoperative atrial fibrillation after cardiac surgery. Chest 2005; 128 (2 Suppl): 24S-7S.

5. Likosky DS, Marrin CA, Caplan LR, et al. Determination of etiologic mechanisms of strokes secondary to coronary artery bypass graft surgery. Stroke 2003; 34: 2830-4. 